\title{
The capabilities of the calculated approach for the astroclimatic assessment in radioastronomy
}

\author{
Nikolay V. Ruzhentsev*, Alexander S. Mihailov \\ Department of Microwave Radiospectrometry, Institute of Radio Astronomy, National Academy of Sciences, Kharkov, Ukraine; \\ *Corresponding Author: ruzh@rian.kharkov.ua
}

Received 30 December 2009; revised 3 February 2010; accepted 25 February 2010.

\begin{abstract}
The work is dedicated to calculation of daily variability of monthly averaged full vertical atmospheric absorption for six well-known mountain locations of sub-millimeter wave band radiotelescopes obtained with usage of chosen by authors models combination. Test locations were defined as follows: Chajnantor plateau in the Atacama mountain desert (Chile), Hanle (India), South Pole (Antarctic), Mauna Kea (Hawaii, USA), Sierra Negra (Puebla, Mexico) and EI Leoncito (Argentine). The data of these calculations were compared with the data of long term radiometric observations of other authors. Searching for new alternative places to complement existing sub-millimeter telescopes locations was attempted too.
\end{abstract}

Keywords: Radiotelescopes; Atmosphere Absorption; Sub-Millimeter Waves Range; Astroclimate

\section{INTRODUCTION}

Earth atmosphere causes considerable impediments for radioastronomical observations at millimeter and submillimeter waves bands due to atmosphere attenuation and instability its transfer function. Consequently, even a slight improvement of the transfer function and its stability can lead to a tangible radioastronomical observations efficiency increase, especially in the submillimeter wave range. In view of this and taking uniqueness and high cost of the radiotelescopes that are installed in various regions of the world, location astroclimatic suitability assessment is required. These assessments are usually experimental and consist of gathering the statistical information concerning the extent of atmospheric attenuation, it's seasonal and daily unsteadiness to reveal the most favorable time and place for the observation to be performed [1-5]. A few only places with most suitable for allocation of sub-millimeter waves radiotelescopes are known [6] to the present time in a world. It is Chajnantor plateau in the Atacama mountain desert (Chile), Hanle in the Chanthang mountain plateau (India), South Pole (Antarctic) Mauna Kea (Hawaii, USA). The unquestionable advantage of the experimental approach is the precise measurement of the atmospheric attenuation in the defined frequency range in the defined location. However, the most significant disadvantages of this approach are the fact that only a limited set of frequencies is used for the experiment (usually 1 or 2 frequencies) [1-4], the necessity of continuous observations cycles that last several years, financial, hardware and organizational efforts and expenses of this important, but yet auxiliary support for radioastronomic observation.

On the contrary, analytical astroclymatic assessment approaches could provide operative and cheap daily and seasonal unsteadiness prognostication for any millimeter and submillimeter wavelength in any location of the world. However, there was an impediment on the route to the practical implementation of this approach: There were no adequate models of global distribution of meteorological parameters altitude profile that were required for this kind of astroclymatic assessment until present days. The purpose of this work is the finding and demonstrating new possibility (unavailable earlier) of obtaining of astroclimatic estimations of atmospheric attenuation average values by calculated method, as well as definition new locations of radio telescopes (most favourable for radioastronomical observations) of submillimetre range.

As we have shown recently in [7-9] (on the example of 15 regions of Ukraine) utilization of the chosen combination of Liebe's atmosphere attenuation model and the latest meteorological atmosphere standard (grounded on the ERA-15 base data) [10-12] allows us to acquire a good (within the $10 \%$ precision interval) harmony of analytical calculations and experimental data concerning the monthly attenuation average in the atmosphere above the plain-type landscape. It is also very important for certain applications that the chosen models combination 
allows the atmospheric absorption variability prognostication for different times of day. However, mentioned above application of models need to be considered for the mountainous landscape also because a great amount of radiotelescopes at millimeter range and especially submillimeter range are located up to $5 \mathrm{~km}$ above the sea level.

This work contains the calculation of daily variability of monthly averaged full vertical atmospheric absorption using the chosen model combination for six well-known mountain locations of submillimeter waverange radiotelescopes. Test locations were defined as follows: Chajnantor plain in the Atacama mountain desert (Chile, $5000 \mathrm{~m}$ ), Hanle (India, $4500 \mathrm{~m}$ ), South Pole (Antarctic, $2800 \mathrm{~m}$ ), Mauna Kea (Hawaii, USA, 4100 m), Sierra Negra (Puebla, Mexico, $4600 \mathrm{~m}$ ) and El Leonsito (Argentina, $2500 \mathrm{~m})$.

Data from these calculations were compared with data of long period radiometric observations (from one year up to nine years) of other authors. Searching for new, alternative or other places to complement existing submillimeter telescope locations was attempted.

\section{RESULTS OF CALCULATIONS AND ITS COMPARISON WITH EXPERIMENTAL DATA}

Figure 1 displays analytically acquired dependencies of monthly average full vertical atmospheric absorption values on the UT time of day for Chajnantor during January and July on the frequency of $225 \mathrm{GHz}$.

International astronomic submillimeter waverange observatory (ALMA) is located on the height of $5 \mathrm{~km}$ above sea level. Nine years cycle atmospheric absorption yearly observation results for the frequency of $225 \mathrm{GHz}$ [1] as well as the results of our calculations are displayed on the same figure.

Figure 2 displays the calculated dependencies of the full vertical atmospheric absorption on the UT time of day for Hanle during January and July on the frequency of $220 \mathrm{GHz}$. Indian astronomic submillimeter waverange observatory (IAO) is located on the height of $4.5 \mathrm{~km}$ above sea level in the Hanle. Atmospheric absorption year cycle observation results for the frequency of 220 $\mathrm{GHz}$ taken from [2] are displayed on the same figure.

Figure 3(a) display analytically acquired dependencies of monthly average full vertical atmospheric absorption values on the UT time of day for EI Leoncito during January, February and July on the frequency of $405 \mathrm{GHz}$. EI Leoncito is an Argentinean-Brazilian sub-millimeter wave range radiotelescope located on the height of $2.5 \mathrm{~km}$ above sea level. The results of one-year cycle of atmospheric absorption observation for 405 $\mathrm{GHz}$ frequency taken from [4] are displayed on the Figure 3(b).
Figure 4(a) display analytically acquired dependencies of monthly average full vertical atmospheric absorption values on the UT time of day for South Pole during January and July on the frequency of $225 \mathrm{GHz}$. American Antarctic remote observatory with a sub-millimeter wave range radiotelescope (AST-RO) was located in the South Pole on the height of $2.8 \mathrm{~km}$ above sea level. The results of half-year cycle of atmospheric absorption observation for the $225 \mathrm{GHz}$ frequency taken from [3] are displayed on the Figure 4(b).

In the Figures 1-4 the rather close layout of data calculated by us and points taken from the literary data [1-5] is well visible for radio telescopes in El Leoncito Argentina $(405 \mathrm{GHz})$, on South Pole $(220 \mathrm{GHz})$, in Hanle (220 GHz), in Chajnantor $(225 \mathrm{GHz})$.

The differences of calculation data and data of experiments, as a rule, are concentrated in the interval of

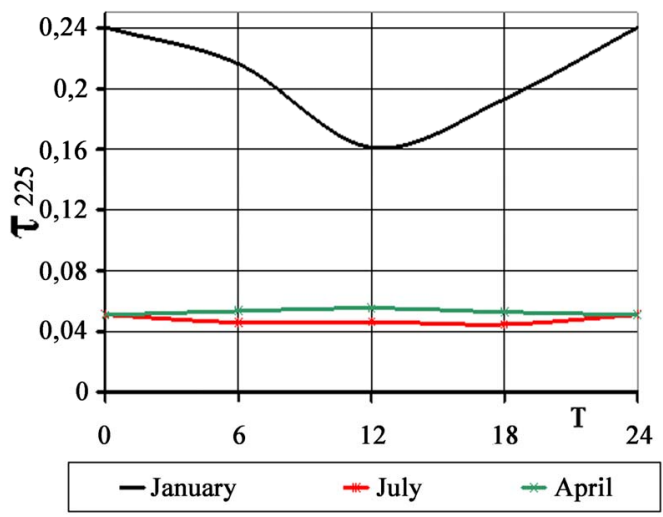

(a)

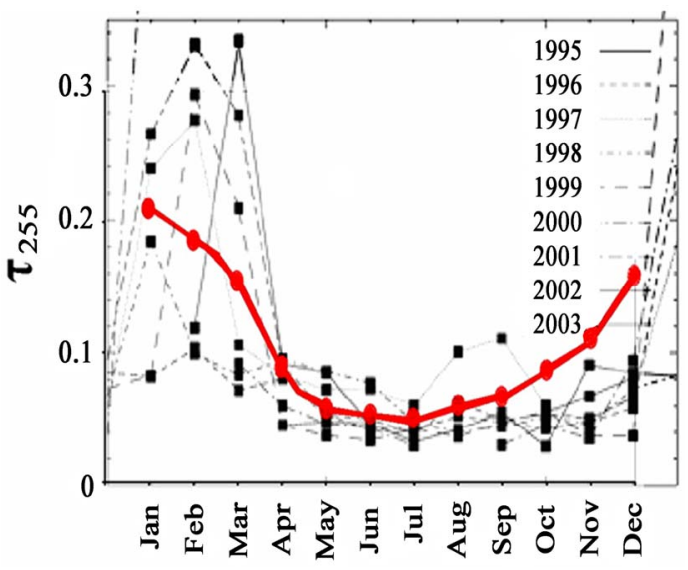

(b)

Figure 1. Calculated dependencies of the daily changing of the monthly average values of full vertical atmospheric absorption (a) as well as the results of our calculations (bold curve) and experimental observations [1] (square) of monthly averaged values of vertical atmospheric absorption (b) for the Chajnantor $(225 \mathrm{GHz})$. 


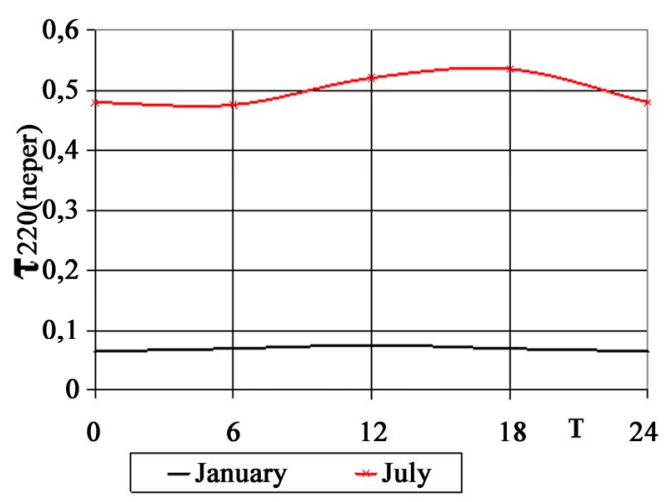

(a)

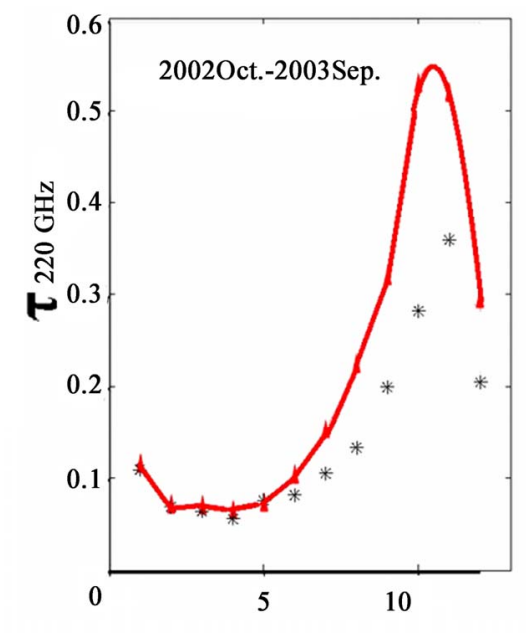

(b)

Figure 2. Calculated dependencies of daily changing of the monthly average values of full vertical atmospheric absorption (a) as well as the results of our calculations (bold curve) and experimental observations [2] (squares) of monthly averaged values of vertical atmospheric absorption (b) for the Hanle on the frequency of $220 \mathrm{GHz}$.

$10-20 \%$ in the winter and $20-30 \%$ in the summer for the year courses of monthly average values of vertical absorption of atmosphere on all of selected by us radiotelescopes. (The separate summer month only takes place an exception in Chajnantor and in Hanle, when these differences are noticeably higher.) Our calculations of diurnal variability have completely qualitatively coinciding with experiment and differed quantitatively from experiments less than on $15-20 \%$.

Let's remark that it would be possible to expect a decreasing all these $10-30 \%$ values of differences in a case of more correct comparison of calculated and experimental data. The absence of complete correctness in realization of such comparisons is caused by different periods of averaging for different literary experimental data and data of our calculation during definition of monthly vertical absorption values of atmosphere. The values of this parameter calculated by us were based on the data of fifteen-years meteorological observations, while the experimental values reduced in the literature were obtained by averaging from radiometer data for one of separated years only.

Influence of above-mentioned cause is well visible, for example, on dispersion of experimental values of monthly average absorption defined for different years in the Chajnantor (Figure 1) or in the Hanle [2]. The small differences (less than 15\%) between data of our calculations (averaged for fifteen-years term) and experimental average-year values of absorption in Mauna Kea [5] (averaged for eleven-years term) as well as between experimental data for Sierra Negra [13] (averaged for four-years term) shows a validity of such point of view.

Besides it is necessary to take into account that the experimental measurements were carried out not always in the conditions of completely clear atmosphere. But nevertheless a carried out qualitative and quantitative (though upper estimations) rating of errors of considered

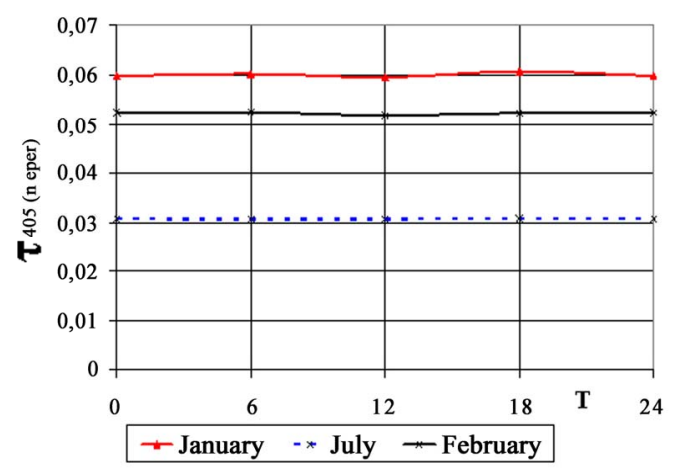

(a)

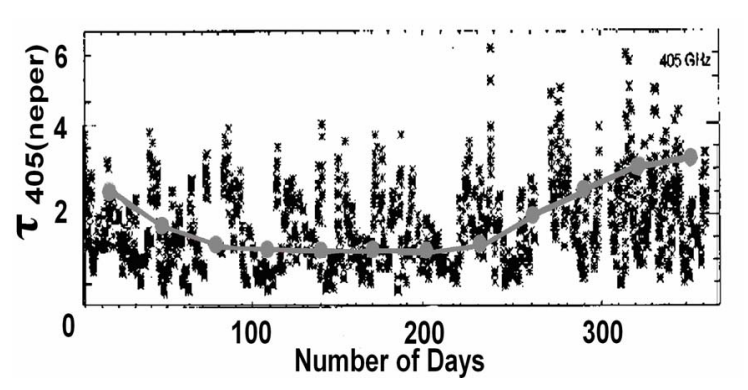

(b)

term of observations: 28 February-1 March

Figure 3. Calculated dependencies of daily changing of the monthly average values of full vertical atmospheric absorption (a) as well as the results of our calculations of monthly averaged (gray curve) and daily experimental observations (squares) values of vertical atmospheric absorption [4] (b) for EI leoncito observatory on $405 \mathrm{GHz}$. 


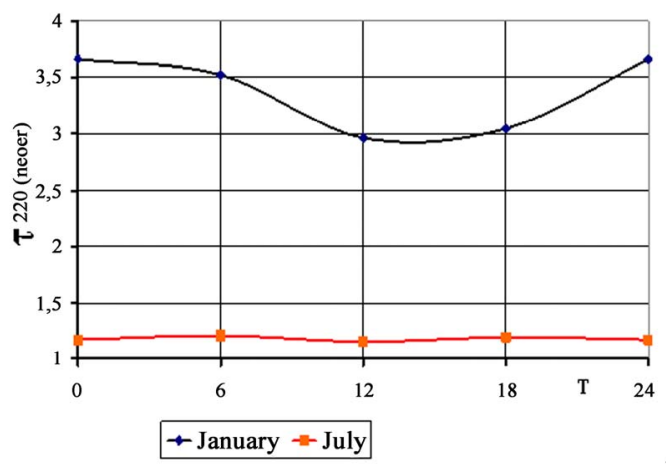

(a)

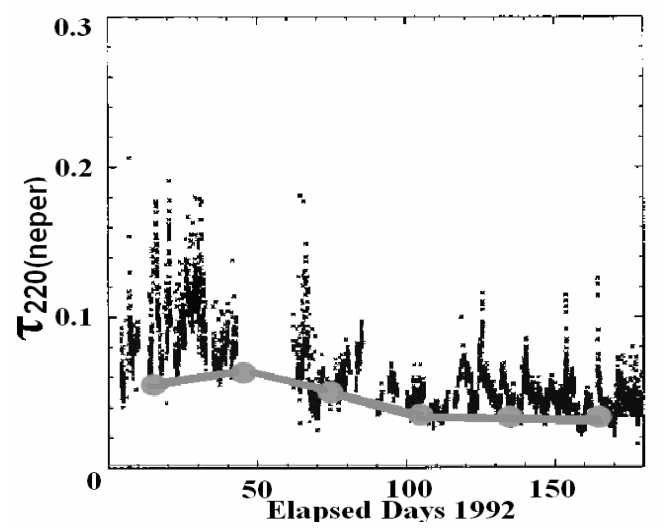

(b)

Figure 4. Calculated dependencies of daily changing of the monthly average values of full vertical atmospheric absorption (a) as well as the results of our calculations of monthly averaged (gray curve) and daily experimental observations (squares) values of vertical atmospheric absorption [3] (b) for South Pole on the frequency of $220 \mathrm{GHz}$.

by us method of astroclimatic forecasting is visual and useful enough (Figures 1-4).

\section{SEARCH FOR ALTERNATIVE AND ADDITIONAL RADIOTELESCOPE LOCATIONS}

The results that were acquired in the previous section demonstrated an efficiency, high operability and functional capabilities of calculational approach to astroclymatic assessment. At the same time, analysis of the most successful locations of operational sub-millimeter wave range radiotelescopes from the astroclymatic point of view [6] allows us to note that out of four radiotelescope locations two are situated in the southern hemisphere (one in the Andes and one in the Antarctic) and two in the tropics of the northern hemisphere (in the Hawaii and in the Indian part of Chanthang mountain plateau).
And the most notable locations of southern hemisphere are distinguished by being more seasonally stable and by a smaller optical thickness value than in the northern hemisphere. According to our calculation (Figure 5) we can note that the best location in the astroclymatic point of view would be southern pole where monthly-average atmospheric absorption values $(\tau)$ are two-three times less than, for example, for the Mauna Kea or in the summer months for the Chajnantor.

Seasonal vertical atmospheric absorption variability is characteristic for every sub-millimeter wave range radiotelescope location, but in different degrees. In connection with that, the best radio astronomical observations from November to April can be made in the South Pole, Hanle and Mauna Kea, while from May to October they can be made on the South Pole, Chajnnator and Mauna Kea (Figure 5).

Mentioned alternative locations or complementary locations (Russian Altay, Rocky Mountains in Colorado, New Earth in Russia, Greenland, Chines Gobi desert, Tibetan Chanthang plateau, etc.) were chosen by us for consideration on the common physical grounds (altitude above sea level, latitude, climate peculiarities). The comparison of result calculations that were carried out for these new places allowed us to distinguish only three astroclymatically suitable locations to compete with best known locations.

These are: Greenland $(\mathrm{H}=2,2 \mathrm{~km}, 80 \mathrm{~N}, 40 \mathrm{~W})$, Chinese part of Chanthang plateau $(\mathrm{H}=5 \mathrm{~km}, 33 \mathrm{~N}, 94 \mathrm{E})$ and Altay $(\mathrm{H}=3 \mathrm{~km}, 50 \mathrm{~N}, 88 \mathrm{E})$ (Figure 6). For example, first two locations are not inferior to and even better than the South Pole and the Hanle from November to April.

It is noticeable that their monthly-average atmospheric absorption value and seasonal variability is almost identical (Figure 5 and Figure 6) between the one in the Chajnantor (ALMA) and found location on the Greenland (if corresponding seasons are compared for northern and southern hemispheres). Moreover, calculations

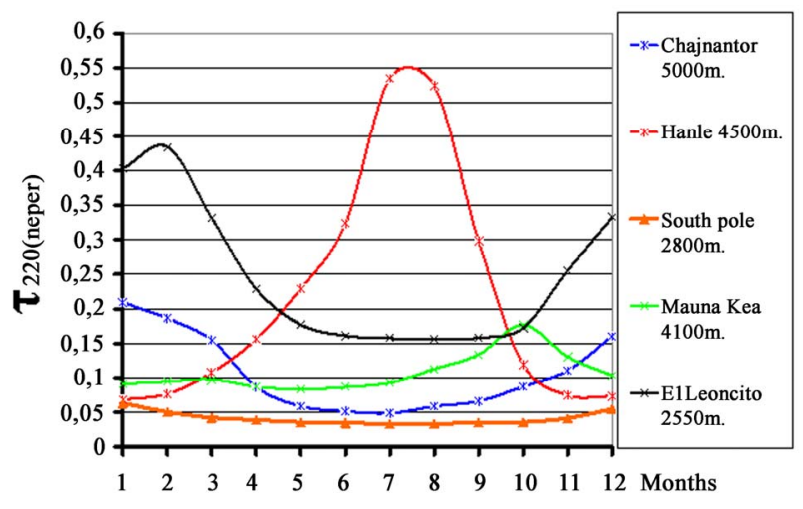

Figure 5. Annual changing $\tau$ for the most known locations of radiotelescopes of sub-millimetre waves. 


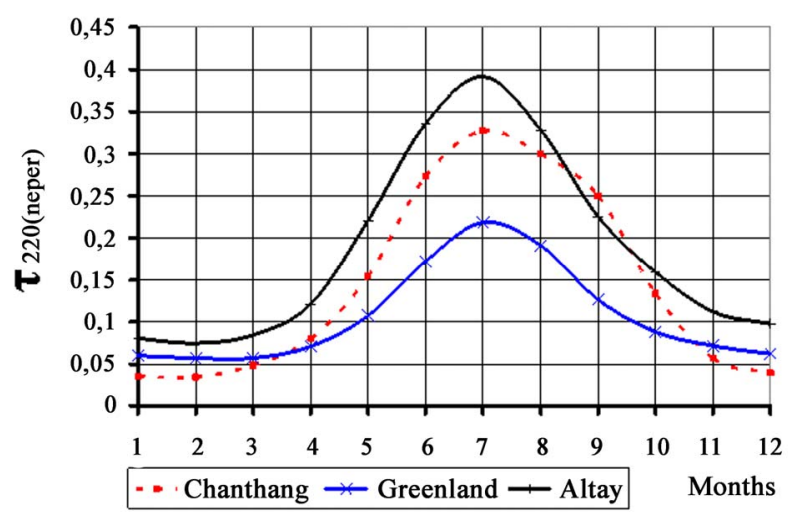

Figure 6. Annual changing $\tau$ for the new found locations which are suitable for radiotelescopes of sub-millimetre waves.

show that optical atmospheric thickness daily deviation is almost absent in the Greenland during all year while this value reaches up to $50 \%$ in summer in the Chajnantor (Figure 1(a)).

Comparing data on Figure 5 and Figure 6 allows us to note noticeable (almost twice) astroclymatic conditions increase while shifting on the Chanthang plateau from Hanle (India, $\mathrm{H}=4.5 \mathrm{~km}, 32 \mathrm{~N}, 78 \mathrm{E}$ ) to Tibetan location $(\mathrm{H}=5 \mathrm{~km}, 33 \mathrm{~N}, 94 \mathrm{E})$ which is situated on the same plateau, but on the Chinese part of it. The same situation is observed while shifting from El Leoncito (Argentine) in the Atakama to the Chajnantor in Chile. However, this case is also affected by the altitude difference of these locations.

\section{CONCLUSIONS}

Thus, for the first time is shown that usage most of modern standard of atmosphere (designed by ESA on the basis of the database ERA-15) in aggregate with MPM model of atmospheric attenuation allows to obtain a seasonal-diurnal statistics of vertical atmospheric absorption for any item of a world. Such possibility of the calculated method was shown as by comparison of the original data of calculation with the experimental data of other authors, as and by definition new astroclimatically favourable locations of sub-millimetre radiotelescopes. This new and previously unavailable ability to obtain astroclymatic assessments of $\tau$ ensures high operability, functional abilities increase and minimal expenditures in comparison with the traditional approach that is based on long-term experimental observations (which usually uses to choose the location of the projected radiotelescope or to specify astroclymatic assessment in the locations of operated radiotelescopes).

\section{REFERENCES}

[1] Simon, J.E. (2003) Radford (NRAO), Conditions for observing with the ALMA at Chajnantor. http://www. tuc. nrao.edu/alma/site

[2] Ananthasubramanian, P.G., Yamamoto, S., Prabhu, T.P. and Angchuk, D. (2004) Measurements of $220 \mathrm{GHz}$ atmospheric transparency at IAO, Hanle, during 20002003. Bulletin of Astronomy Society in India, 32(2), 99111.

[3] Richard, A., Chamberlin and Bally, J. (1994) 225-GHz atmospheric opacity of the South Pole sky derived from continual radiometric measurements of the sky-brightness temperature. Applied Optics, 33(6), 1095-1099.

[4] Melo, A., Kaufmann, P., de Castro, C., Raulin, J., Levato, H., Marun, A., Giuliani, J. and Pereyra, P. (2005) Submillimetre-wave atmospheric transmission at El Leoncito, Argentina Andes. IEEE Transactions, AP-53(4), 15281534.

[5] Masson, C. (1990) Atmospheric opacity and water vapor. Sub-Millimetre Array Technical Memorandum, 12(1), 10-14.

[6] http://en.wikipedia.org/wiki/Submillimetre astronomy

[7] Mihailov, A.S. and Ruzhentsev, N.V. (2007) Features of global allocation of atmospheric attenuation in the range 10-1000 GHz. Radiophysics and Radio Astronomy, in Russian, 12(1), 76-83.

[8] Mihailov, A.S. and Ruzhentsev, N.V. (2009) Research of spatial distribution of atmospheric attenuation for territory of Ukraine at millimetre-waves band. Applied Radioelectronics, in Russian, 2(1), 12-22.

[9] Ruzhentsev, N.V., Mihailov, A.S. and Shirin, A.M. (2007) Investigations of season-diurnal dependencies of atmospheric absorption with usage of model ERA-15 and its additional testing. Proceedings of Eeleventh URSI Commission F Open Symposium on Radio Waves Propagation and Remote Sensing, Rio de Janeiro, RS3.3-1-RS3.3-5.

[10] Liebe, H.J. (1989) MPM-An atmosphere millimeter wave propagation model. International Journal on Infrared and Millimeter Waves, 10(6), 631-650.

[11] Martellucci, A., Rastburg, B.A., Poiares Baptista, J.P.V. and Blarzino, G. (2003) New reference standard atmospheres based on numerical weather products. Abstracts of International Workshop-ClimDiff'2003, Fortaleza, clim.1.

[12] Riva, C., Martellucci, A., Kubista, E., Chonhuber, M. and Luini, L. (2005) ERA-15 climatological databases for propagation modeling. Proceedings of International Conference-ClimDiff, Cleveland, 26-27 September 2005, clim. 12.1-12.7.

[13] The Large Millimeter Telescope (LMT) site: http:www. $\underline{\text { lmtgtm.org/site.html }}$ 